\title{
Imaging Characteristics of Breast Lymphoma; a Case Series
}

\author{
Sara Rehman, Muhammad Atif Naveed, Javaria Aleem \\ Department of Radiology, Shaukat Khanum Memorial Cancer Hospital and Research Centre, Lahore, \\ Pakistan
}

Received: 03 November 2019/Accepted: 27 December 2019

\section{OPEn ACCESS}

\section{Correspondence:}

Dr. Sara Rehman, Department of Radiology, Shaukat Khanum Memorial Cancer Hospital and Research Centre, Lahore, Pakistan. Email: sararehman_88@ yahoo.com

Citation: Rehman S, Naveed MA, Aleem J. Imaging characteristics of breast lymphoma; a case series. J Cancer Allied Spec [Internet]. 2020 Jan.6; 6(1):e1002957. https://doi. org/10.37029/jcas.v6i1.305

Copyright: (c) 2020 Rehman S, Naveed MA, Aleem J. This is an open access article distributed under the terms of the Creative Commons Attribution License, which permits unrestricted use, distribution, and reproduction in any medium, provided the original author and source are credited.

Funding: Nil.

Competing interests: Nil.

\begin{abstract}
Introduction: Breast involvement by lymphoma is rare. It can occur as a primary breast tumour or as an extranodal manifestation of the systemic disease. The imaging features of breast lymphoma (BL) are not characteristic. Biopsy is necessary for diagnosis due to non-specific imaging features. Materials and Methods: A retrospective electronic medical chart review was conducted of patients diagnosed with lymphoma of breast that underwent diagnostic radiological procedures (including mammography, ultrasound breast, computed tomography (CT) scan and positron emission tomography (PET/CT) scan from 1 July 2018 to 31 March 2019 at Shaukat Khanum Memorial Cancer Hospital and Research Centre, Pakistan. Results: Four patients were identified. On mammogram, the most common finding consisted of the presence of high-density masses with circumscribed or indistinct margins. On ultrasound, hypoechoic masses and indistinct diffuse infiltrative patterns were observed. PET/CT and CT were helpful in detecting extramammary sites of disease and for classifying the disease into primary or secondary BL. Conclusion: The early diagnosis of the $B L$ is important. The radiologists should include lymphoma in the differential diagnosis when there is the absence of microcalcifications or spiculated margins on mammography and ultrasound.
\end{abstract}

Key words: Breast lymphoma, case series, mammogram, positron emission tomography/computed tomography imaging, ultrasound

\section{Introduction}

Breast is an uncommon site for haematological malignancies. Lymphoma is the most common haematological malignancy affecting the breast. The incidence of breast lymphoma (BL) is approximately $0.04 \%-0.70 \%$ of all breast malignancies. ${ }^{[1]}$ Secondary forms of the disease are more common than primary disease. Nonetheless, primary BL (PBL) accounts for $0.05 \%-0.53 \%$ of all breast region cancers. ${ }^{[2,3]}$

$B L$ can present with several non-specific symptoms clinically, such as painless or palpable breast mass(es). Occasionally, nipple retraction or discharge and skin changes may also occur, which may mimic primary breast carcinoma. ${ }^{[2,4]} \mathrm{PBL}$ usually presents early as palpable breast mass and as a solitary mass on imaging. ${ }^{[2]}$ In comparison, secondary $B L(S B L)$ affects older age group with usually a non-specific occult clinical presentation, multiplicity on imaging and simultaneous disease in the breast and other visceral organs. ${ }^{[3]}$

There is only handful of studies which have focused on the radiological features of $\mathrm{BL}$, and they show that there are no pathognomonic features of $B L^{[3,4]}$ 
To the best of our knowledge, no investigation has been carried out to assess the imaging features of $B L$ in our population. The aim of the present investigation was to evaluate the radiological features of $B L$ in this region.

\section{Materials and Methods}

A retrospective electronic medical chart review was conducted of patients that were diagnosed with $B L$ and had underwent diagnostic radiological procedures including mammography, ultrasound breast, computed tomography (CT) scan or positron emission tomography (PET/CT) from 1 July 2018 to 31 March 2019 at Shaukat Khanum Memorial Cancer Hospital and Research Centre, Pakistan. Breast cancers of other aetiology or BL patient without any diagnostic radiological investigation were excluded from the study. This study was approved by the Institutional Review Board of Shaukat Khanum Memorial Cancer Hospital and Research Centre (04-07-19-01).

The investigators deidentified the medical data for all participants. Information regarding the participants' demographic data, age, medical history, diagnosis, pathological reports and radiological studies were extracted.

All participants underwent a combination of imaging modalities. These consisted of ultrasound breast, mammograms, CT scan of chest, abdomen and pelvis with contrast and PET/CT. The decision on the type of imaging was based on the clinical presentation, available resources and results of prior investigations. Ultrasound imaging was performed for breasts using $7.5 \mathrm{MHz}$ linear array transducer Toshiba Aplio XG. Breast mammograms were performed bilaterally on dedicated mammographic equipment; Senographe Essential (GE), which was using to acquire standard mediolateral oblique and craniocaudal views. Baseline CT scan of chest, abdomen and pelvis with intravenous contrast was carried on Prime SP Aquilion scanner (160 slice), after administration of $1.5-1.67 \mathrm{ml} / \mathrm{kg}$ of iodinated intravenous contrast medium at a rate of $1.5-2 \mathrm{ml} / \mathrm{s}$ by a Medrad Salient injector with a scan delay of $70 \mathrm{~s}$ after onset of injection. Typical imaging parameters were $120 \mathrm{kVp}, 150 \mathrm{mAs}$ and $5 \mathrm{~mm}$ slice thickness with a pitch of 1.2. PET/CT was as performed by administering $5 \mathrm{MBq} / \mathrm{kg}$ body weight (F-18)-fluorodeoxyglucose intravenously. Imaging was performed on an integrated 16 slice Gemini EF PET/CT scanner (Philips), with scanning from head to thigh. The subsequent CT scan was performed with intravenous contrast material, using parameters and equipment as described before.

The diagnosis of $B L$ was made based on the histopathological findings of ultrasound-guided biopsy. Classification into primary and secondary lymphoma was done on the basis of radiological findings on CT scan or PET/CT as per Wiseman and Lao criteria for PBL [Table 1]..5] All patients were discussed in multidisciplinary team (MDT) meeting and underwent follow-up evaluation. The response of treatment was assessed according to the response evaluation criteria in solid tumours (RECIST) criteria. ${ }^{[6]}$

\section{Results}

\section{Case 1}

A 60-year-old woman presented with the history of the left breast lump for 1 month. There was no history of fever, night sweats or chills. On physical breast examination, there was a large firm, nontender mass in upper outer quadrant of the left breast and palpable ipsilateral axillary lymph nodes.

On mammogram, the left breast showed two adjacently placed circumscribed high-density masses in the upper outer quadrant, in the anterior

Table 1: Criteria for primary breast lymphoma as per Wiseman and Liao. ${ }^{[5]}$

1. The availability of adequate pathology material

2. Both mammary tissue and lymphomatous infiltrate are present

3. No widespread disease or preceding extramammary lymphoma

4. Ipsilateral axillary node involvement is considered acceptable 
breast. There were no suspicious calcifications, but enlarged ipsilateral axillary lymph nodes were seen [Figure 1.1a and b]. Her breast ultrasound demonstrated a heterogeneous hypoechoic mass in the upper outer quadrant corresponding to mammogram findings [Figure 1.2a] and a similarappearing mass at distance of $6 \mathrm{~mm}$ from the aforementioned mass [Figure 1.2b]. Her left axilla showed few enlarged lymph nodes with partially effaced fatty hilum [Figure 1.2c]. Ultrasoundguided biopsy was performed and it confirmed the diagnosis of diffuse large B-cell lymphoma (DLBCL).

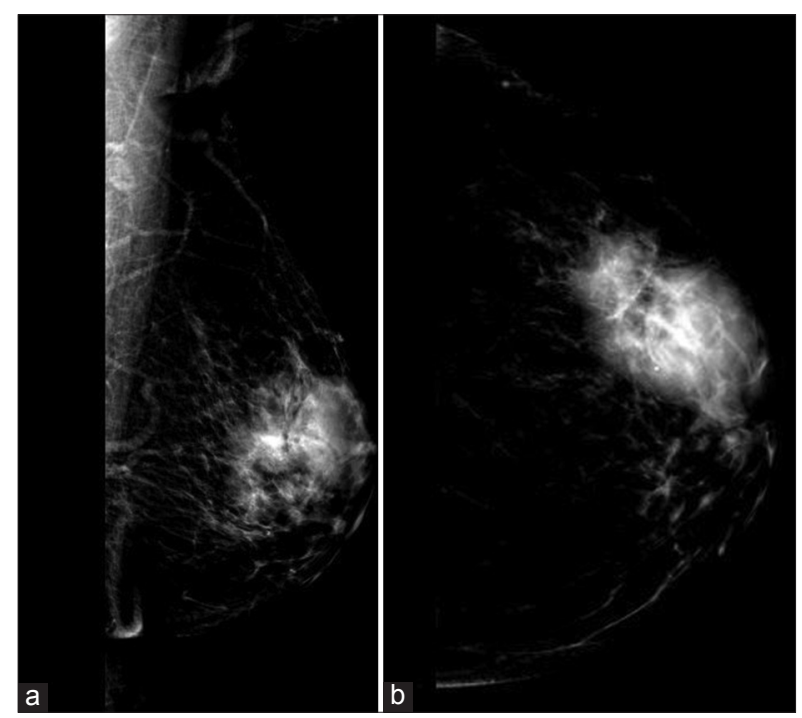

Figure 1.1: ( $a$ and b) Mediolateral oblique and craniocaudal views of the left breast show two adjacently placed high-density masses with circumscribed margins in upper outer quadrant in anterior breast. No microcalcifications
Ultrasound-guided fine-needle aspiration cytology for the left axilla showed reactive lymph node. A bone marrow biopsy was also performed that was negative for disease involvement. Her PET/CT scan revealed increased metabolic activity in both masses in the left breast and a standardised uptake value of 10.1 [Figure 1.3a and b]. Furthermore, there was increased metabolic activity in ipsilateral axillary and mediastinal lymph nodes with no metabolic activity elsewhere to suggest metastatic disease. The presence of mediastinal hypermetabolic activity was in favour of SBL.

She received 6 cycles of chemotherapy with rituximab, cyclophosphamide, adriamycin, vincristine and prednisone (R-CHOP) with central nervous system (CNS) prophylaxis, consisting of 2 cycles of high-dose intrathecal methotrexate (MTX) as per MDT discussion. On a follow-up CT scan, there was an interval partial treatment response according to RECIST criteria. ${ }^{[6]}$

\section{Case 2}

A 34-year-old woman presented with the left breast lump for 4 months and headache. There was no history of fever, night sweats or chills. On local breast examination, there were palpable masses in both breasts with no axillary lymphadenopathy.

Ultrasound of breast was performed. The left breast demonstrated a circumscribed hypoechoic mass corresponding to palpable site, extending in all
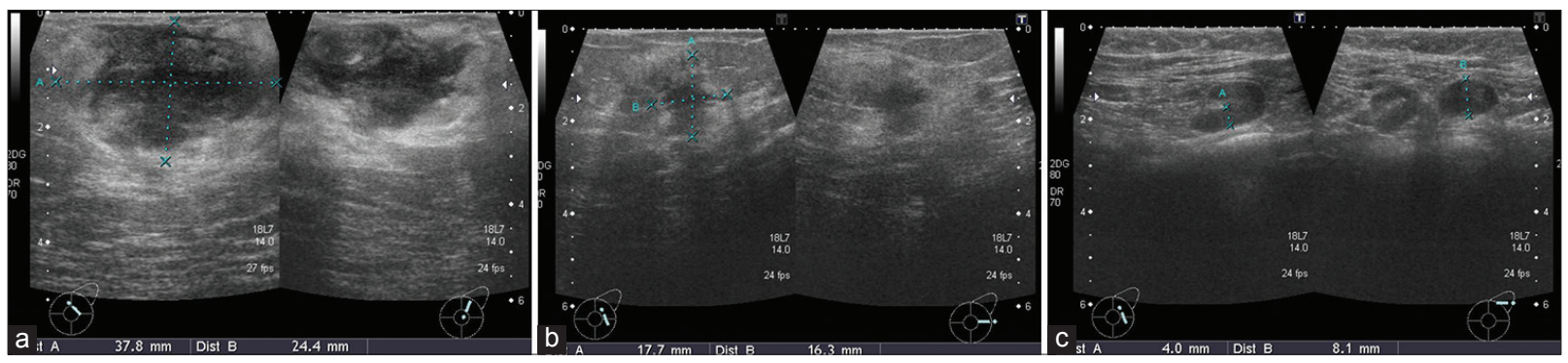

Figure 1.2: $(a$ and $b$ ) Left breast ultrasound shows two adjacently placed masses in the left breast 2 and 3 o'clock, measuring $38 \mathrm{~mm} \times 24 \mathrm{~mm}$ and $17 \mathrm{~mm} \times 16 \mathrm{~mm}$, respectively, with interlesional distance of $6 \mathrm{~mm}$ and demonstrating heterogeneous echotexture that gives 'pseudocystic' appearance. (c) Enlarged left axillary lymph nodes with partially effaced fatty hila, measuring $4 \mathrm{~mm}$ and $8 \mathrm{~mm}$, respectively 
breast quadrants. In addition, a couple of satellite masses were found [Figure 2.1 $a$ and b]. No enlarged axillary nodes were present. Ultrasound of the right breast revealed an indistinct, hypoechoic mass in the central right breast. There was no evidence of increased vascularity on the Doppler ultrasound interrogation of the mass. However, few satellite masses [Figure 2.1c and d] and enlarged ipsilateral axillary lymph nodes were appreciated. Ultrasound-
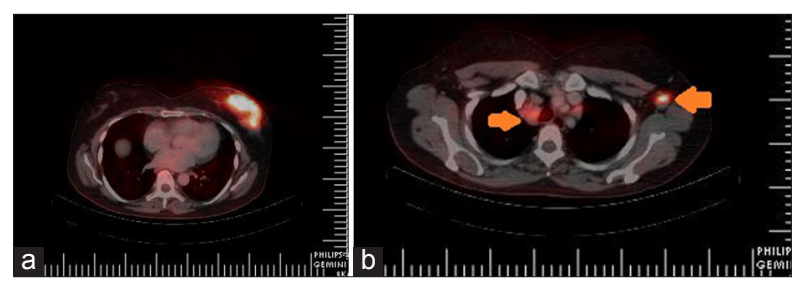

Figure 1.3: ( $a$ and b) Fused positron emission tomography/computed tomography image is showing increased fluorodeoxyglucose uptake in the mass in the left breast with maximum standardised uptake value of 10.1 along with uptake in ipsilateral axillary and right paratracheal lymph nodes guided biopsy was performed for the right breast retroareolar main mass and satellite mass at 6 o'clock and left breast main mass and largest satellite mass. The histopathology of biopsy samples confirmed the diagnosis of bilateral breast DLBCL. Subsequently, the contrast-enhanced CT scan showed a large enhancing mass with central low density in both breasts along with enlarged enhancing axillary nodes [Figure 2.2]. There was no evidence of visceral or nodal metastasis above or below diaphragm; therefore, this case was diagnosed as PBL.

MDT decision was to start chemotherapy with 6 cycles of R-CHOP and 2 cycles of high-dose intrathecal MTX for CNS prophylaxis, followed by radiation therapy (XRT) to breasts. When she was undergoing her $1^{\text {st }}$ cycle of chemotherapy, she had sudden cardiac arrest. Multiple resuscitation attempts were made and she was shifted to intensive care unit (ICU) for further management. The patient experienced ischaemic brain injury
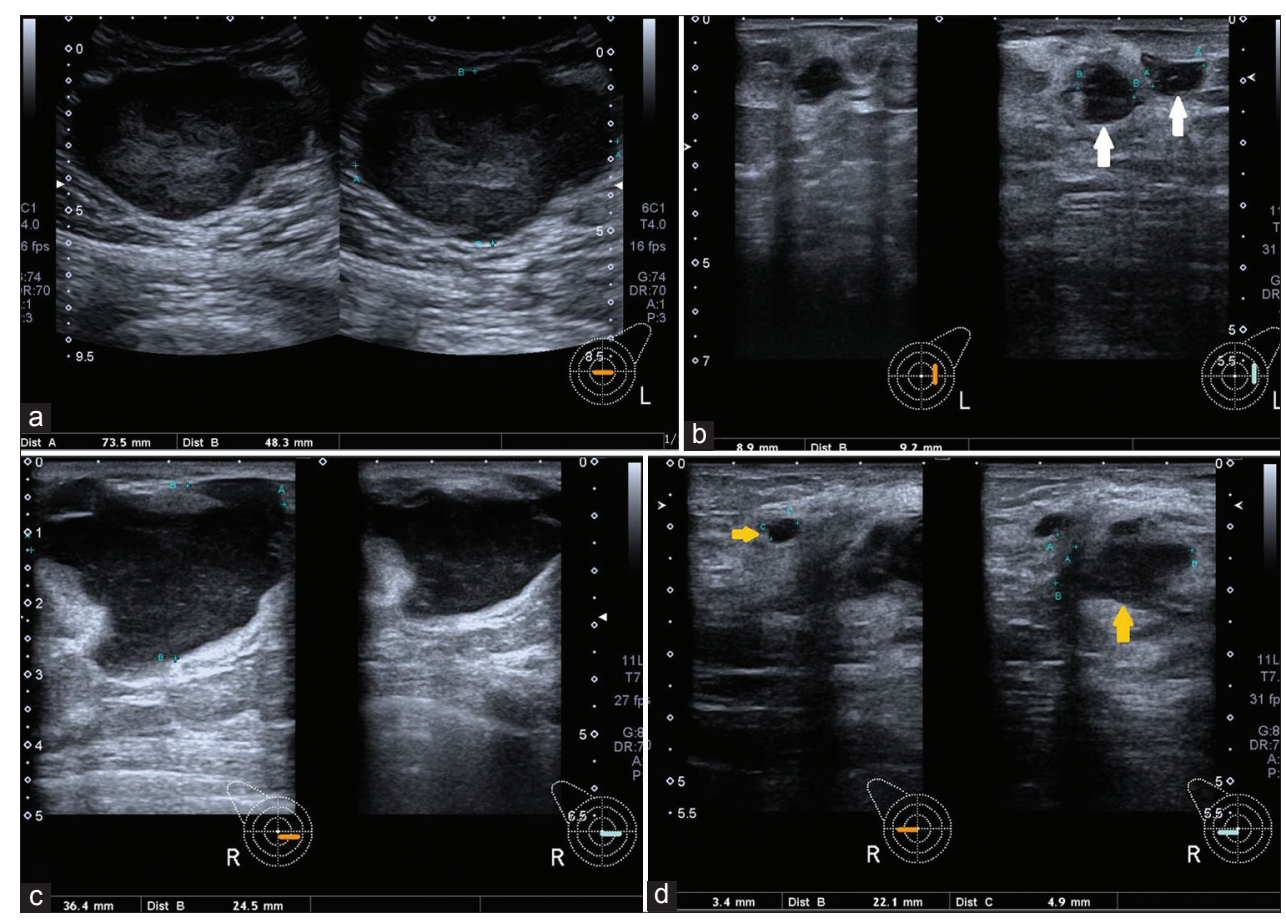

Figure 2.1: (a) Ultrasound of the left breast is showing a large heterogeneous hypoechoic retroareolar mass, measuring $7.3 \mathrm{~cm} \times 4.8 \mathrm{~cm}$. (b) Couple of rounds to oval hypoechoic satellite nodules (white arrows). (c) Ultrasound of the right breast is showing poorly circumscribed hypoechoic mass at 3 o'clock with retroareolar extension, measuring $3.6 \mathrm{~cm}$ $\times 2.5 \mathrm{~cm}$. (d) Hypoechoic satellite lesions (yellow arrows) 
and died after prolonged ICU admission of approximately 75 days.

\section{Case 3}

A 61-year-old woman presented with the left breast lump for 6 months. There was a history of high-grade fever associated with rigors, chills, body aches and generalised weakness for 1 month. She had multiple comorbid conditions; including uncontrolled diabetes mellitus (Type II) and chronic kidney disease, stage 4.

Mammogram demonstrated a high-density mass with indistinct margins in the left breast which was present on the upper outer quadrant and upper central aspect, extending into the axillary tail [Figure 3.1 $a$ and b]. There were no suspicious grouped calcifications; however, benign linear calcifications were noted in the left breast. The ipsilateral axillary nodes were enlarged. The mammogram findings were confirmed on breast ultrasound, which showed an indistinct hypoechoic mass, extending from 12 o'clock to 2 o'clock as well as into the axillary tail [Figure 3.2a and b]. There were multiple enlarged ipsilateral axillary lymph nodes identified with loss of fatty hila. Ultrasound-guided biopsies were performed for the left breast mass and left axillary lymph node. The histopathology examination revealed DLBCL and reactive lymphoid hyperplasia, respectively.

CT neck to pelvis revealed more aggressive disease. There was a huge paramediastinal soft tissue mass in the right haemithorax which was invading the adjacent upper and middle lobes of the right lung. Mediastinal and pericardial invasion was present with superior vena cava compression and associated pleural effusion. Osseous involvement of the right $4^{\text {th }}$ rib was also demonstrated. The left breast mass was irregular and it was involving the overlying skin and extending into axilla and pectoralis major muscles. In addition, there was splenomegaly [Figure 3.3]. Based on the widespread disease in baseline $C T$, she was diagnosed as a patient of SBL.

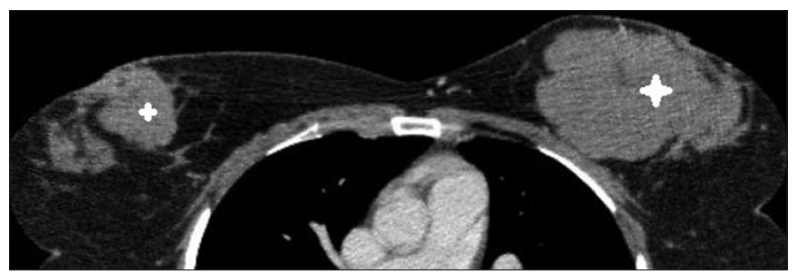

Figure 2.2: Axial computed tomography image of chest in soft tissue window shows heterogeneously enhancing circumscribed masses with indistinct margins in both breasts parenchyma with surrounding fat stranding
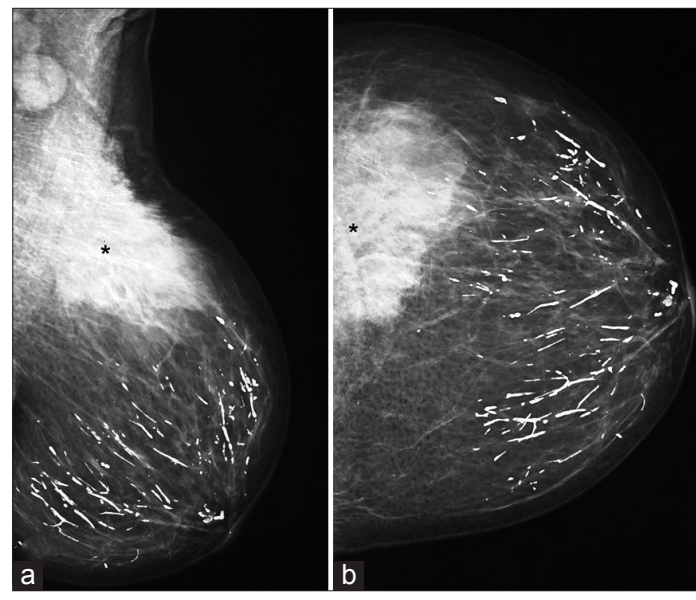

Figure 3.1: ( $a$ and $b$ ) Mammogram mediolateral oblique (a) and craniocaudal views (b) demonstrate a high-density mass with indistinct margins $\left(^{*}\right)$ in the left breast, upper outer quadrant and upper central aspect, extending into the axillary tail

Considering multiple comorbid conditions, MDT recommendation was to initiate palliative care, as she was unlikely to tolerate chemotherapy-associated toxicity. This patient was then lost to follow-up.

\section{Case 4}

A 32-year-old female presented with the left breast lump for 5 weeks. There was no history of fever, night sweats or chills. She underwent a biopsy of this mass and histopathology result was suggestive of DLBCL. Her CT chest, abdomen and pelvis showed a large left breast mass, occupying the lateral quadrants and enlarged left axillary, external iliac and pelvic sidewall lymph nodes [Figure 4.1 a]. Furthermore, the CT was significant for a soft tissue mass in the left ischiorectal fossa, 

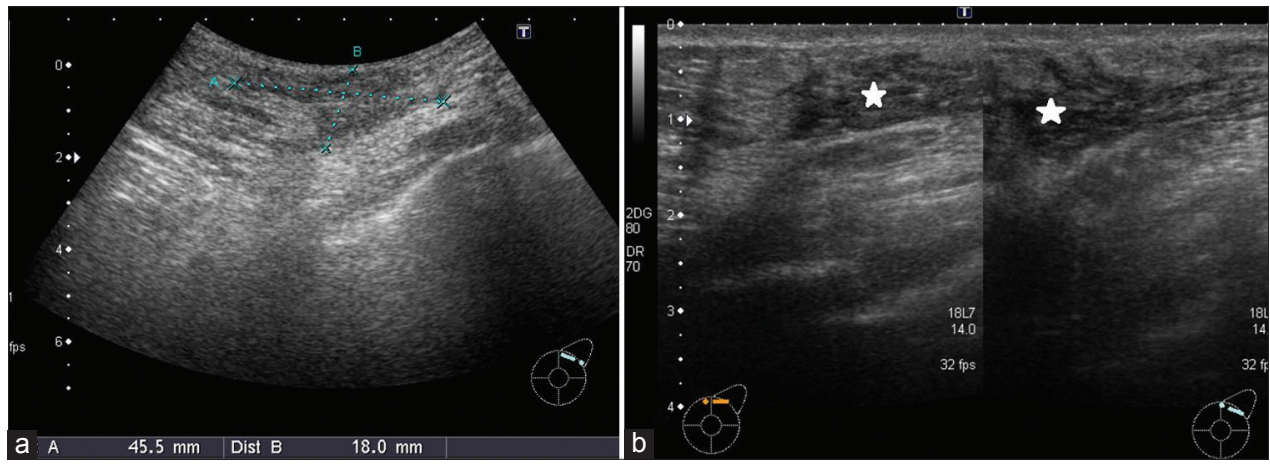

Figure 3.2: ( $a$ and b): Gray scale ultrasound demonstrates an irregular, solid mass with indistinct margins and heterogeneous echo pattern, measuring $45 \mathrm{~mm} \times 18 \mathrm{~mm}$ (white star). It is associated with overlying skin infiltration and thickening

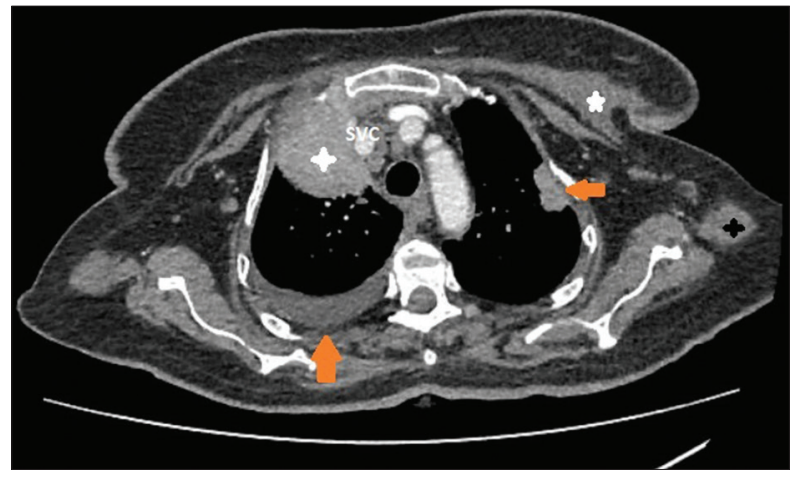

Figure 3.3: Contrast-enhanced axial computed tomography image of thorax in soft tissue window shows aggressive disseminated disease. Large right paramediastinal locally infiltrative soft tissue heterogeneously enhancing mass is encasing and compressing the superior vena cava (white star). Left pleural based soft tissue nodule and right pleural effusion (orange arrows). Left breast deep infiltrative mass (white star) along with enlarged enhancing axillary lymph node (black star)

which was concerning for disease involvement [Figure 4.1b]. Subsequently, she underwent a CT-guided biopsy of ischiorectal fossa mass and it showed the same histopathology, thereby, confirming the diagnosis of SBL.

MDT recommendations were to start chemotherapy with 6 cycles of R-CHOP with 2 cycles of high-dose intrathecal MTX for CNS prophylaxis and consolidative XRT to breast due to initial bulky disease. A follow-up CT scan was done after 3 cycles of chemotherapy which showed minimal hypodense linear soft tissue in the upper outer quadrant of the left breast and complete resolution of previously documented pelvic lymphadenopathy and left ischiorectal fossa mass. Four months after completion of her chemotherapy, the patient underwent PET/CT. Based on RECIST criteria, the patient showed a good treatment response with interval resolution of the left breast soft tissue mass along with metabolically insignificant residual soft tissue thickening [Figure 4.2]. The patient then received XRT of the left breast (200 centigray in 18 fractions) as per initial MDT instructions.

\section{Discussion}

Haematological malignancies are rare in breast. Nonetheless, lymphoma is the most common haematological malignancy to affect the breast. ${ }^{[1,3]}$ The clinical presentation of BL is non-specific and may consist of a solitary or multiple unilateral or bilateral painless palpable breast masses, nipple or skin retraction and nipple discharge. On the contrary, the usual clinical symptoms seen with lymphomas such as fever, weight loss and night sweats, are very rare in PBL. The most common histologic type in BL is DLBCL. Other types, such as follicular lymphoma, mucosa-associated lymphoid tissue lymphoma or Burkett lymphoma are found in minority of cases. ${ }^{[7,8]}$ The differential diagnosis of $\mathrm{BL}$ includes phyllodes tumour, atypical fibroadenoma, breast hamartoma, primary epithelial carcinoma and metastasis. ${ }^{[7]}$ 


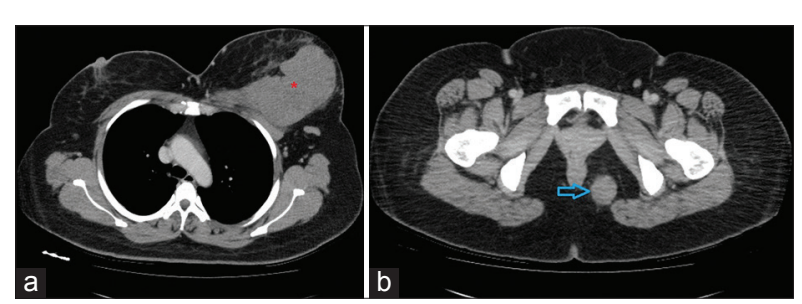

Figure 4.1: (a) Contrast-enhanced axial computed tomography (CT) image of the thorax shows large left breast mass, measuring $10.7 \mathrm{~cm} \times 10 \mathrm{~cm}\left(\mathrm{red}^{\star}\right)$, occupying the lateral quadrants and enlarged left axillary lymph node, measuring $2 \mathrm{~cm}$ in long axis. (b) Contrastenhanced axial CT image of the pelvis shows soft tissue mass seen in the left ischiorectal fossa, measuring $3.3 \mathrm{~cm} \times 2.8 \mathrm{~cm}$ (blue arrow)

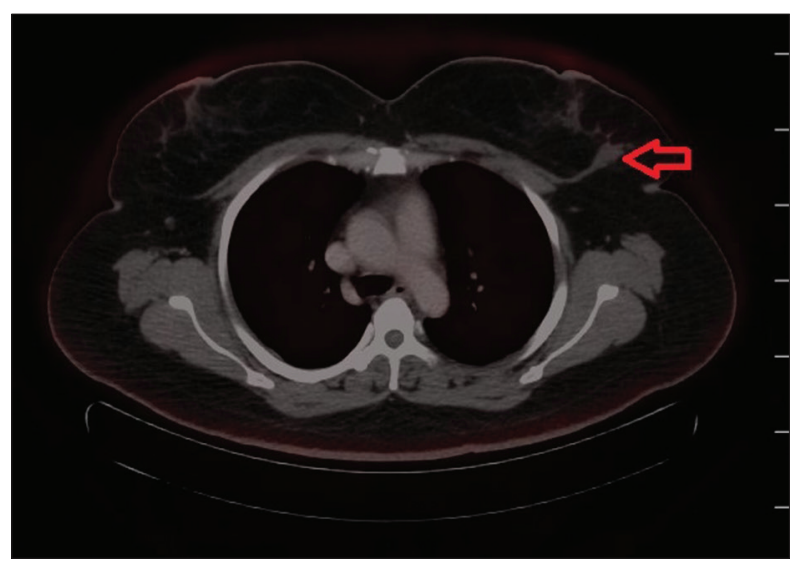

Figure 4.2: Fused positron emission tomography/ computed tomography shows interval resolution of the left breast soft tissue mass with metabolically insignificant residual soft tissue thickening (red arrow)

PBL has female predominance with most commonly unilateral presentation. Bilateral involvement occurs in $1 \%-14 \%$ of cases. ${ }^{[9]}$ It has been suggested that mammogram and ultrasound breast are required to evaluate breast mass and axillary lymph nodes. ${ }^{[4]}$ Typically, on mammogram, PBL presents as an oval-shaped solitary mass with irregular or indistinct margins, preserved parenchymal architectural pattern without microcalcifications or spiculated margins. ${ }^{[10]}$ It may present with diffuse increase in breast density. Appearance of axillary lymphadenopathy is rare. The SBL has variable imaging features on mammogram and ultrasound; however, multiplicity, extramammary extension and nodal involvement are favourable findings. ${ }^{[4]}$ In a case series by Liberman et al. of 32 cases of nonHodgkin lymphoma in 29 women (66\% classified as $\mathrm{PBL}$ ), the most common mammographic finding was a solitary mass without calcifications (69\%). Other findings consisted of multiple masses (9\%) and diffuse increased opacity with skin thickening (9\%). Interestingly, no mammographic abnormality was reported in 13\% of the cases. ${ }^{[11]}$ In another retrospective review of 36 patients with $\mathrm{BL}$ ( 22 primary and 14 secondary) by Surov, no significant differences were noted on mammography and sonography between primary and SBL. ${ }^{[4]}$ Similarly, mammographic findings in our cases were increased density masses, with well-circumscribed margins in one patient, while indistinct margins in the other case. Typical features which are frequently seen in invasive epithelial tumours such as spiculated margins or suspicious microcalcifications were not present.

As in mammography, ultrasound lacks characteristic features. Ultrasound features of $B L$ range from hypoechoic or isoechoic echotexture to diffuse infiltrative appearance with distinct or indistinct margins. Increased echogenicity, mixed hypo- and isoechoic pattern or pseudocystic appearance have also been described in literature. ${ }^{[4,6,11,12]}$ Posterior acoustic enhancement can be present with an echogenic rim or onion peel-like rim surrounding the mass. ${ }^{[3]}$ Lymphomas are usually hyper-vascular on Doppler ultrasound. Our cases demonstrated comparable findings as mentioned in literature. Two of the patients showed hypoechoic masses with circumscribed margins in one patient and indistinct margins in other case. Indistinct diffuse infiltrative pattern was also observed in a case.

CT study of chest, abdomen and pelvis can be useful for the evaluation of the local extent, invasion of chest wall, extramammary spread of disease and nodal involvement in the thorax or below the diaphragm. In the present case series, three patients underwent CT scan. The BL masses appeared as a mass with circumscribed 
or irregular margins, with moderate or increased enhancement. This is similar to that reported previously. ${ }^{[4]}$

CT and PET/CT detect extramammary sites of disease involvement and aid in classification of $B L$ (PBL or $\mathrm{SBL}$ ). PET/CT scan performed as baseline study in one case showed hypermetabolic activity in the breast mass and mediastinal and axillary lymph nodes and thus detecting metastatic involvement. PET/CT performed as follow-up imaging after chemotherapy showed the treatment response in the form of decrease in tumour size and metabolic activity. These findings are similar to those described by Yang et al. ${ }^{[3]}$ It has an acceptable role in staging, evaluation of treatment response and detection of recurrence on follow-up. ${ }^{[7,8]}$ None of our participants underwent magnetic resonance imaging (MRI) of breast(s). There are handful of studies that have described MRI features of $B L .{ }^{[4,9]} \mathrm{BL}$ appears as an iso to hypointense mass on T1-weighted images and heterogeneously hyperintense on T2-weighted images, with marked early heterogeneous enhancement after gadolinium administration. Kinetics curves demonstrate a rapid early enhancement and plateau (Type II) or a slow washout in the delayed phase (Type III) curve..$^{[4,7]}$

A possible limitation of the present investigation is that this was a retrospective analysis. As such, it was not possible to have a uniform set of standardised imaging modalities of all participants. Nonetheless, in this investigation, we have presented a diverse set of imaging modalities and described their findings in great details. Another limitation is that this series only consists of four participants. BL is a rare disease and it was not possible to include more participants. Furthermore, this is a novel case series, as to best of our understanding, no similar investigation has yet been published regarding imaging features of $B L$ in this region. Based on the findings from this study, further investigations can be carried out to identify and assess patients of BL.
The early diagnosis of the $B L$ is extremely relevant. Non-specific clinical presentation and imaging features require clinical history review, multimodality imaging approach and histopathology for diagnosis and management. Nonetheless, the radiologists should include lymphoma in the differential diagnosis when there is the absence of microcalcifications or spiculated margins on mammography and sonography. PET/CT finds its role in staging as well as in follow-up.

\section{Conflicts of Interest}

The authors declare that there are no conflicts of interest.

\section{References}

1. Shim E, Song SE, Seo BK, Kim YS, Son GS. Lymphoma affecting the breast: A pictorial review of multimodal imaging findings. J Breast Cancer 2013;16:254-65.

2. Sabaté JM, Gómez A, Torrubia S, Camins A, Roson N, De Las Heras P, et al. Lymphoma of the breast: Clinical and radiologic features with pathologic correlation in 28 patients. Breast J 2002;8:294-304.

3. Yang WT, Lane DL, Le-Petross HT, Abruzzo LV, Macapinlac HA. Breast lymphoma: Imaging findings of 32 tumors in 27 patients. Radiology 2007;245:692-702.

4. Surov A, Holzhausen HJ, Wienke A, Schmidt J, Thomssen C, Arnold D, et al. Primary and secondary breast lymphoma: Prevalence, clinical signs and radiological features. Br J Radiol 2012;85:e195-205.

5. Wiseman C, Liao KT. Primary lymphoma of the breast. Cancer 1972;29:1705-12.

6. Nishino M, Jagannathan JP, Ramaiya NH, Van den Abbeele AD. Revised RECIST guideline version 1.1: What oncologists want to know and what radiologists need to know. AJR Am J Roentgenol 2010;195:281-9.

7. Irshad A, Ackerman SJ, Pope TL, Moses CK, Rumboldt T, Panzegrau B. Rare breast lesions: Correlation of imaging and histologic features with WHO classification. Radiographics 2008;28:1399-414.

8. Lyou CY, Yang SK, Choe DH, Lee BH, Kim KH. Mammographic and sonographic findings of primary breast lymphoma. Clin Imaging 2007;31:234-8.

9. Maurya V, Singh G, Ram B, Ganguli P. Multimodality imaging features of primary breast lymphoma a rare entity. Indian J Radiol Imaging 2019;29:85-8.

10. Liberman L, Giess CS, Dershaw DD, Louie DC, 
Deutch BM. Non-Hodgkin lymphoma of the breast: Imaging characteristics and correlation with histopathologic findings. Radiology 1994;192:157-60.

11. Kumar R, Xiu Y, Dhurairaj T, Yu JQ, Alavi A, Zhuang H. F-18 FDG positron emission tomography in non-Hodgkin lymphoma of the breast. Clin Nucl Med 2005;30:246-8.

12. Jabbour G, El-Mabrok G, Al-Thani H, El-Menyar A, Al Hijji I, Napaki S. Primary breast lymphoma in a woman: A case report and review of the literature. Am J Case Rep 2016;17:97-103.

\section{Authorship Contributions}

Conceived and designed the analysis; SR, collected the data; SR and JA, contributed data or analysis tools; SR, MAN and JA, performed the analysis; N/A, and wrote the paper; SR, MAN and JA. 